\title{
Diffusion Monte Carlo Calculations for Rare-earths: Applying the Long-range Corrected Scheme to Minnesota M06 Functional
}

\author{
Nagat Elkahwagy, $^{1, *}$, Atif Ismail ${ }^{1,2}$, S. M. A. Maize $^{3}$, K. R. Mahmoud ${ }^{1}$ \\ ${ }^{1}$ Physics Department, Faculty of Science, Kafrelsheikh University, Egypt \\ ${ }^{2}$ Physics Department, Faculty of Applied Sciences, Umm al-Qura University, Saudi Arabia \\ ${ }^{3}$ Physics Department, Faculty of Science, Menoufia University, Egypt
}

Copyright $\mathrm{C} 2016$ by authors, all rights reserved. Authors agree that this article remains permanently open access under the terms of the Creative Commons Attribution License 4.0 International License

\begin{abstract}
By employing the diffusion Monte Carlo (DMC) method, we test the success of the unpublished long-range corrected M06 (LC-M06) functional, compared to LC-BLYP functional, in improving the calculations of the ground and excited states energies for some $4 \mathrm{f}$ - and $5 \mathrm{f}$-rare-earths at different values of the range separation parameter $\mu$. It is found that LC-M06 functional performs well for both the ground and the excited states of 4f-lanthanides. In addition, our results reveal that the optimal value of $\mu$ for the ground state lies between $0.40-0.50$ a.u. while a smaller optimal value $\mu=0.35$ a.u. is found for the excited states. However, LC-BLYP ground and excited states results at the optimal value of $\mu$ seem to be the best for 5 f-actinides.
\end{abstract}

Keywords Diffusion Monte Carlo, Minnesota Functional, Rare-earths

\section{Introduction}

A computational study of rare-earths has received much attention in the past few decades. In fact, several problems have probably obstructed an accurate calculation for these elements. One major difficulty is the strong electronic correlations which should not be neglected in accurate calculations.

Among the most successful methods in treating the strongly correlated electrons systems is the diffusion Monte Carlo (DMC) method which explicitly includes electron-electron correlation effects. Moreover, it scales well with system size, with the computational cost increasing as the cube of the number of electrons.

In order to arrive to chemical accuracy, methods such as density functional theory (DFT) have been used as a practical way of constructing the determinantal part of the wavefunction instead of Hartree-Fock. Conventional hybrid functionals especially the most popular B3LYP functional proved to be a promising tool in many papers [1-3]. But despite its success, the conventional hybrid functionals (which combine a pure exchange functional in DFT with HF exchange integral in a constant ratio) are not usually regarded as a fundamental approach to solve all DFT problems probably since these functionals contains only short-range HF exchange so the long-range asymptotic behavior still remains incorrect. The introduction of long-range HF exchange in DFT functional will correct the description of long-range exchange interaction. So the long-range corrected functionals which are originally developed by Gill [4] and Savin [5-7] are providing a considerable improvement with respect to the hybrid functionals. The general form of the long-range

$$
\begin{aligned}
& E_{x c}{ }^{L C-D F T}=E_{c}{ }^{D F T}+\left(1-C_{H F}\right) E_{x}{ }^{S R-D F T}+ \\
& C_{H F} E_{x}{ }^{S R-H F}+E_{x}{ }^{L R-H F}
\end{aligned}
$$

where the traditional exchange-correlation functional $E_{x c}$ may be a pure or hybrid functional. The components labeled "LR" and "SR" are evaluated using the long-range and short-range Coulomb potentials, respectively, while $C_{H F}$ denotes the amount of HF exchange present in the original functional.

Very recently we have tested the performance of the standard B3LYP and the long-range corrected LC-BLYP functionals for both the ground and the excited states of lanthanides and actinides [8]. Our calculations have indicated that both functionals are not suited for 4f-lanthanides which have strongly localized nature. However, the most popular B3LYP functional has given reasonable results for 5 f-actinides. Furthermore, applying the long-range correction to BLYP functional has clearly enhanced both the ground and the excited states for 5 f-actinides.

In this paper, we examine the performance of LC-M06 functional for the ground and excited states of some 
rare-earths at different values of the range separation parameter $\mu$. To our knowledge, it is the first time that combines the long-range corrected scheme with the hybrid M06 functional. The basic form of the wave function that we used is the Slater-Jastrow wave function which is considered the most common and simplest one. The rest of this paper is organized as follow: In the next section, we briefly describe the DMC method. Then we present and discuss the results. Finally, we give our conclusion.

\section{Computational Methods}

Diffusion Monte Carlo method has been extensively described in the literatures [9-11] so we give here a brief description of it. The diffusion Monte Carlo (DMC) method is a stochastic projector method for solving the imaginary time many-body Schrödinger equation

$$
-\frac{\partial}{\partial \tau} \psi(R, \tau)=\left(-\frac{1}{2} \nabla^{2}+V-E_{T}\right) \psi(R, \tau)
$$

where $\tau$ is the imaginary time, $\tau=$ it and $E_{T}$ is the energy offset.

Importance sampling with a trial wavefunction $\psi_{T}(R)$ is used to improve the statistical accuracy of the simulation and this is can be achieved by multiplying Eq. (2) by $\psi_{T}(R)$ and rearranging

$$
\begin{aligned}
& -\frac{\partial f(R, \tau)}{\partial \tau}=-\frac{1}{2} \nabla^{2} f(R, \tau)+\nabla \cdot\left[f(R, \tau) \cdot v_{D}(R)\right]+ \\
& +\left[E_{L}-E_{T}\right] f(R, \tau)
\end{aligned}
$$

where $\quad f(R, \tau)=\psi(R, \tau) \psi_{T}(R) \quad$ interpreted as a probability density and $E_{L}(R) \frac{\hat{H} \psi_{T}(R)}{\psi_{T}(R)}$ is the local energy.

this equation can be simulated with a random walk having diffusion, a draft, and a branching step and may be written in the integral form

$$
f(R, \tau+\Delta \tau)=\int G\left(R, R^{\prime} ; \Delta \tau\right) \cdot f(R, \tau) d R
$$

where the Green's function $G\left(R, R^{\prime} ; \Delta \tau\right)$ is a solution of the same equation (3) initial and can be interpreted as a probability of transition from a state $\mathrm{R}$ to $\mathrm{R}$ '. It is possible to use MC method to solve the integral in Eq. (4) but the difficulty is that the precise form of $G\left(R, R^{\prime} ; \Delta \tau\right)$ is not known. Fortunately the comparison of the Schrödinger equation with the diffusion equation gives us a clue about how one might approximate the unknown Green's function.

The evolution during the long time interval $\tau$ can be generated repeating a large number of short time steps $\tau$. In the limit $\tau \rightarrow 0$, one can make use of the short time approximation for Green's function [12]:

$$
\begin{aligned}
& G\left(R, R^{\prime} ; \Delta \tau\right) \approx(2 \pi \Delta \tau)^{-3 N / 2} \exp \left[-\frac{\left(R-R^{\prime}-\Delta \tau \nabla \ln \left|\psi_{T}\right|^{2}\right)^{2}}{2 \Delta \tau}\right] \\
& \exp \left[-\frac{\Delta \tau\left(E_{L}\left(R^{\prime}\right)+E_{L}(R)-2 E_{T}\right)}{2}\right]
\end{aligned}
$$

But due to the fermionic nature of electrons, the wavefunction must have positive and negative parts and this is opposite with the assumed nature of $\psi$ which is a probability distribution. So the fixed-node approximation [13] had been used to deal with the fermionic antisymmetry which constrains the nodal surface of $\psi$ to equal that of the antisymmetric trial wavefunction $\psi_{T}$.

In this work, we start by generating the wavefunction using the quantum chemistry program Gamess [14]. We make use of CRENBL ECP basis set [15] for all elements except for La atom where CRENBS ECP basis set is being used which proved to be successful for the calculations. Both the ground and excited states energies have been calculated within a DMC framework. All QMC computations are performed within Qwalk code [16]. Through all the DMC calculations a time step of $\tau=0.001 H^{-1}$ and a mean population of 2000 walkers are used.

\section{Results and Discussion}

In tables 1 and 2 we list the ground and excited states energies for some lanthanides and actinides respectively calculated using the hybrid M06 functional and the long-range corrected LC-M06 functional at different values of the range separation parameter $\mu$ ranging from 0.30 a.u. to 0.50 a.u. The electronic excitation involves a promotion of an electron with a change of spin from $6 \mathrm{~s} \rightarrow 5 \mathrm{~d}$ and $7 \mathrm{~s} \rightarrow 6 \mathrm{~d}$ for lanthanides and actinides respectively. The values calculated within the standard B3LYP functional for both lanthanides and actinides along with the best values calculated by employing LC-BLYP functional from our earlier work [8] are also involved for comparison.

It is obvious from table 1 that for the atom not having f-electrons, La, the most popular B3LYP seems to be the best choice. M06 functional gives very poor results compared to B3LYP. Although applying the long-range corrected scheme to M06 functional improves the quality of the results, the conventional B3LYP is still the best. On the other hand, for $4 \mathrm{f}$-lanthanides the performance of M06 functional is also poor. However, it is found that LC-M06 functional enhances considerably the ground state energies at a value of range separation parameter around $\mu=0.40-0.50$ a.u. for all of $4 \mathrm{f}$-lanthanides. However, except for $\mathrm{Sm}$, the optimal value of $\mu$ for the energy of excited states is found to be at a smaller value $\mu=0.35$ a.u. On the contrary, applying the long-range corrected scheme to BLYP functional does not improve the results for $4 \mathrm{f}$-lanthanides as revealed in our previous study [8]. 
Table 1. Ground state energies $G$ and excited state energies $E$ (due to 6s-5d transition) computed within DMC for lanthanides using LC-M06 functional as a function of $\mu$ compared to B3LYP and M06 functionals.

\begin{tabular}{lcccccccc}
\hline \hline Method & State & $\mathrm{La}$ & $\mathrm{Ce}$ & $\mathrm{Pr}$ & $\mathrm{Nd}$ & $\mathrm{Pm}$ & $\mathrm{Sm}$ & $\mathrm{Eu}$ \\
\hline B3LYP $^{\mathrm{s}}$ & $\mathrm{G}$ & -1.2556 & -2.7138 & -4.7310 & -9.3288 & -14.3620 & -19.7039 & -28.6630 \\
& $\mathrm{E}$ & -1.2407 & -2.5894 & -5.3237 & -9.3609 & -14.0353 & -20.2920 & -28.7529 \\
$\mathrm{M} 06$ & $\mathrm{G}$ & -1.2149 & -2.6600 & -5.5325 & -8.9997 & -14.3154 & -20.7858 & -28.6905 \\
& $\mathrm{E}$ & -1.2092 & -4.8685 & -5.3717 & -8.8680 & -14.2145 & -20.8146 & -28.5964 \\
LC-M06 & & & & & & & & \\
$\mu=0.30$ & $\mathrm{G}$ & -1.2536 & -2.7297 & -5.5767 & -9.2908 & -14.3970 & -20.7371 & -28.6068 \\
& $\mathrm{E}$ & -1.2430 & -2.7751 & -5.5446 & -9.2294 & -14.3146 & -20.3199 & -28.6013 \\
$\mu=0.35$ & $\mathrm{G}$ & -1.2517 & -2.7257 & -5.4540 & -9.3153 & -14.3868 & -19.8996 & -28.6575 \\
& $\mathrm{E}$ & -1.2430 & -2.8537 & -5.9366 & -9.2812 & -14.4187 & -19.3141 & -28.7573 \\
$\mu=0.40$ & $\mathrm{G}$ & -1.2554 & -2.9456 & -5.5356 & -9.3253 & -14.4060 & -20.7711 & -28.6225 \\
& $\mathrm{E}$ & -1.2430 & -2.7097 & -5.7081 & -9.2497 & -14.2927 & -20.4593 & -28.6764 \\
$\mu=0.45$ & $\mathrm{G}$ & -1.2092 & -2.7128 & -5.9090 & -9.2920 & -14.3951 & -20.8158 & -28.7001 \\
& $\mathrm{E}$ & -1.2048 & -2.8541 & -5.4788 & -9.2474 & -14.2288 & -20.3744 & -28.6363 \\
$\mu=0.50$ & $\mathrm{G}$ & -1.2320 & -3.1441 & -5.6387 & -9.3024 & -14.3949 & -20.8077 & -28.6498 \\
& $\mathrm{E}$ & -1.2411 & -2.7040 & -5.4406 & -9.2332 & -14.3302 & -20.6674 & -28.6232 \\
\hline \hline
\end{tabular}

a refers to ref. 8

Table 2. Ground state energies $G$ and excited state energies $E$ (due to 7s-6d transition) computed within DMC for actinides LC-M06 functional as a function of $\mu$ compared to B3LYP, M06 and LC-BLYP functionals.

\begin{tabular}{ccccccccc}
\hline \hline Method & State & $\mathrm{Ac}$ & $\mathrm{Th}$ & $\mathrm{Pa}$ & $\mathrm{U}$ & $\mathrm{Np}$ & $\mathrm{Pu}$ & $\mathrm{Am}$ \\
\hline B3LYP $^{\mathrm{a}}$ & $\mathrm{G}$ & -29.4865 & -35.6253 & -42.6491 & -51.2060 & -60.5301 & -71.3357 & -74.5900 \\
& $\mathrm{E}$ & -29.4374 & -35.5777 & -42.5859 & -51.1979 & -60.4908 & -71.3974 & -74.8191 \\
LC-BLYP & & & & & & & & \\
$\mu=0.35$ & $\mathrm{G}$ & -29.4749 & -35.6445 & -42.6880 & -51.3280 & -60.6486 & -71.4135 & -74.7796 \\
& $\mathrm{E}$ & -29.3970 & -35.5769 & -41.9985 & -51.2047 & -60.6103 & -71.2421 & -74.8217 \\
$\mu=0.40$ & $\mathrm{G}$ & -29.4787 & -35.6510 & -42.6774 & -51.2510 & -60.5861 & -71.4043 & -74.6294 \\
& $\mathrm{E}$ & -29.3778 & -35.5817 & -42.7063 & -51.1696 & -60.3880 & -71.2819 & -74.3282 \\
& & & & & & & & \\
M06 & $\mathrm{G}$ & -29.4749 & -35.6118 & -42.7546 & -51.3074 & -53.4227 & -54.8674 & -74.3488 \\
& $\mathrm{E}$ & -29.4575 & -35.5724 & -42.7316 & -51.1787 & -55.9839 & -56.3098 & -74.3180 \\
LC-M06 & & & & & & & & \\
$\mu=0.30$ & $\mathrm{G}$ & -29.4817 & -35.6224 & -42.6249 & -51.1893 & -60.4764 & -71.4186 & -74.5768 \\
& $\mathrm{E}$ & -29.3951 & -35.5686 & -42.6904 & -51.1701 & -60.5494 & -71.3279 & -74.7598 \\
$\mu=0.35$ & $\mathrm{G}$ & -29.4940 & -35.6429 & -42.6380 & -51.2701 & -60.4601 & -71.3082 & -74.4792 \\
& $\mathrm{E}$ & -29.4344 & -35.5261 & -42.6793 & -51.2045 & -60.4819 & -71.1188 & -74.8201 \\
$\mu=0.40$ & $\mathrm{G}$ & -29.4364 & -35.6406 & -42.7320 & -51.1971 & -60.4667 & -71.3694 & -74.6000 \\
& $\mathrm{E}$ & -29.4294 & -35.5460 & -42.6787 & -51.1395 & -60.4223 & -71.1210 & -74.8265 \\
$\mu=0.45$ & $\mathrm{G}$ & -29.4504 & -35.6267 & -42.6863 & -51.2840 & -60.3924 & -71.2773 & -74.6628 \\
& $\mathrm{E}$ & -29.4486 & -35.5186 & -42.6774 & -51.1772 & -60.5009 & -71.2130 & -74.5943 \\
$\mu=0.50$ & $\mathrm{G}$ & -29.4669 & -35.6512 & -42.6742 & -51.2011 & -60.5317 & -71.2555 & -74.7925 \\
& $\mathrm{E}$ & -29.4374 & -35.5553 & -42.7088 & -51.1510 & -60.4045 & -71.3009 & -74.5975 \\
\hline \hline
\end{tabular}


On the other hand, our calculations show that for Ac and $\mathrm{Th}$, actinide elements with no $5 \mathrm{f}$ electrons, nearly the same energies results either by using LC-M06 or LC-BLYP and no improvement has been observed when compared to B3LYP functional. Also it is obvious that both the ground and the excited states energies are not change significantly by altering the values of the range separation parameter. Whereas when $5 \mathrm{f}$-subshells are being populated, both the ground and the excited states are altered considerably. This emphasizes that atoms in which the f-subshells are not occupied are less affected by the introduction of long-range $\mathrm{HF}$ exchange than atoms having electrons in there f-subshells. It is also interested to note that at the beginning of $5 \mathrm{f}$-actinides, M06 functional gives reasonable results but the results become very poor by increasing the number of 5 f-electrons across the series.

For most of $5 \mathrm{f}$-actinides, the ground state energies are improved at $\eta$ in the range $0.45-0.50$ a.u while the optimal value for the excited states is found to be in the range $0.30-0.35$ a.u. It is indicated that these values are different from the optimal values $\mu=0.35$ a.u. and $\mu=0.35-0.40$ a.u. obtained for the ground and the excited states respectively by using LC-BLYP in our previous study. But on the whole, the long-range corrected LC-M06 performs as well as or worse than LC-BLYP functional for 5 f-actinides and the latter two functionals are better than the standard B3LYP.

In view of these results, we can safely note that the choice of $\mu$ not only depends on the system of interest, but also on the type of the functional. Besides, and in accord with Rohrdanz and Herbert [17], the optimal value of the range separation parameter is considerably different for the ground and excited state properties even in the same system.

\section{Conclusions}

To summarize, we have applied the long-range corrected scheme to Minnesota M06 functional and have investigated the performance of the resulting LC-M06 functional for ground and excited states of some rare-earths. The ground and excited states energies have been calculated within a DMC framework. Our results indicate that LC-M06 functional works much better than either LC-BLYP or B3LYP functionals for 4f-lanthanides. However, for 5 f-actinides LC-BLYP results at the optimal value of $\mu$ are still the best.

\section{Acknowledgements}

The authors gratefully thank Dr. Lucas Wagner for helpful discussions in Qwalk quantum Monte Carlo software.

\section{REFERENCES}

[1] L. Wagner and L. Mitas. A quantum Monte Carlo study of electron correlation in transition metal oxygen molecules,
Chem. Phys. Lett. Vol. 370 412, 2003.

[2] E. Sola, J. P. Brodholt, and D. Alfè. Equation of state of hexagonal closed packed iron under Earth's core conditions from quantum Monte Carlo calculations, Phys. Rev. B Vol. 79024107,2009

[3] Shi-Guo. Quantum Monte Carlo Studies of Ultra-Cold Molecules and Rashba Interactions, Ph. D. Thesis, University of North California State, 2014.

[4] P. M. W. Gill, R. D. Adamson, and J. A. Pople. Coulomb-attenuated exchange energy density functionals, Mol. Phys. Vol. 88, No. 4, 1005-1010, 1996.

[5] A. Savin, in Recent Developments and Applications of Modern Density Functional Theory, edited by J. M. Seminario, Elsevier, Amsterdam, 1996.

[6] T. Leininger, H. Stoll, H. J. Werner, and A. Savin. Combining long-range configuration interaction with short-range density functionals, Chem. Phys. Lett. Vol. 275, 151-160, 1997.

[7] J. Toulouse, F. Collona, and A. Savin. Long-rangeshort-range separation of the electron-electron interaction in density-functional theory, Phys. Rev. A, Vol. 70, 062505, 2004.

[8] Nagat Elkahwagy, Atif Ismail, S. M. A. Maize, K. R. Mahmoud. Diffusion Monte Carlo calculations for rare-earths: Hartree-Fock, hybrid B3LYP, and long-range corrected LC-BLYP functional, Univers. J. Phys. Appl., Vol. 10, No. 1, 5-10, 2016.

[9] J. B. Anderson. Quantum Monte Carlo. Origins, Development, Applications, Oxford University Press, 2007.

[10] M. P. Nightingale and C. J. Umrigar, Quantum Monte Carlo methods in physics and chemistry, Kluwer Academic Publishers, 1999.

[11] L. M. Sobol. A primer for the Monte Carlo Method, CRC Press, 1994.

[12] W. M. C. Foulkes, L. Mitas, R. J. Needs and G. Rajagopal. Quantum Monte Carlo simulations of solids, Rev. Mod. Phys. Vol. 73 33, 2001.

[13] J. B. Anderson., Quantum chemistry by random walk. $\mathrm{H}^{2} \mathrm{P}$, $\mathrm{H}_{3}^{+} \mathrm{D}_{3 \mathrm{~h}}{ }^{1} \mathrm{~A}_{1}^{1}, \mathrm{H}_{2}{ }^{3} \Sigma^{+}{ }_{\mathrm{u}}, \mathrm{H}_{4}{ }^{1} \Sigma_{\mathrm{g}}^{+}$, be ${ }^{1} \mathrm{SJ}$, Chem. Phys. Vol. 65 4121, 1976 .

[14] M. W. Schmidt, J. A. Boatz, K. K. Baldridge, S. T. Elbert, M. S. Gordon, J. H. Jensen, S. Koseki, N. Matsunaga, K. A. Nguyen, S. Su, T. L. windus, M. Dupuis, and J. A. Montgomery. General atomic and molecular electronic structure system, J. Comp. Chem. Vol. 14 1347, 1993.

[15] D. Feller. The Role of Databases in Support of Computational Chemistry Calculations, J. Comp. Chem. Vol. 17 1571, 1996.

[16] L. K. Wagner, M. Bajdich, and L. Mitas. QWalk: A Quantum Monte Carlo Program for Electronic Structure, J. Comp. Phys. Vol. 228 3390, 2009.

[17] Mary A. Rohrdanz, Katie M. Martins and John M. Herbert. A long-range corrected density functional that performs well for both ground-state properties and time- dependent density functional theory excitation energies, including charge transfer excited states, J. Chem. Phys. Vol. 130 054112, 2009. 